\title{
Hamiltonian Chains in Hypergraphs
}

\author{
Gyula Y. Katona ${ }^{\dagger}$ \\ Department of Computer Science and Information Theory, \\ Budapest University of Technology and Economics, \\ 1521, P. O. B.: 91, Hungary \\ kiskat@cs.bme.hu
}

Hamiltionian chain is a generalisation of hamiltonian cycles for hypergraphs. Among the several possible ways of generalisations this is probably the most strong one, it requires the strongest structure. Since there are many interesting questions about hamiltonian cycles in graphs, we can try to answer these questions for hypergraphs, too. In the present article we give a survey on results about such questions.

Keywords: Hamiltonian cycle, hypergraph

\section{Introduction}

Let $\mathcal{H}$ be a $r$-uniform hypergraph on the vertex set $V(\mathcal{H})=\left\{v_{1}, v_{2}, \ldots, v_{n}\right\}$ where $n>r$. For simplicity of notation $v_{n+x}$ with $x \geq 0$ denotes the same vertex as $v_{x}$ (unless stated otherwise). The set of the edges, $r$-element subsets of $V$, is denoted by $\mathcal{E}(\mathcal{H})=\left\{E_{1}, E_{2}, \ldots, E_{m}\right\}$. We will write simply $V$ for $V(\mathcal{H})$ and $\mathcal{E}$ for $\mathcal{E}(\mathcal{H})$ if no confusion can arise.

In Katona and Kierstead (1999) the authors defined the notion of a hamiltonian-chain.

Definition 1 A cyclic ordering $\left(v_{1}, v_{2}, \ldots, v_{n}\right)$ of the vertex set is called a Hamiltionian chain iff for each $1 \leq i \leq n \quad\left\{v_{i}, v_{i+1}, \ldots, v_{i+r-1}\right\}=: E_{j}$ is an edge of $\mathcal{H}$. An ordering $\left(v_{1}, v_{2}, \ldots, v_{l+1}\right)$ of a subset of the vertex set is called an open chain of length $l$ between $v_{1}$ and $v_{l+1}$ iff for each $1 \leq i \leq l-r+2$ there exists an edge $E_{j}$ of $\mathcal{H}$ such that $\left\{v_{i}, v_{i+1}, \ldots, v_{i+r-1}\right\}=E_{j}$. An open chain of length $n-1$ is an open hamiltonian chain. A cyclic ordering $\left(v_{1}, v_{2}, \ldots, v_{l}\right)$ of a subset of the vertex set is called a chain of length $l$ iff for every $1 \leq i \leq l$ there exists an edge $E_{j}$ of $\mathcal{H}$ such that $\left\{v_{i}, v_{i+1}, \ldots, v_{i+r-1}\right\}=E_{j}$. (Now $v_{l+x}$ denotes the same vertex as $v_{x}$ ).

Since an ordinary graph is a 2-uniform hypergraph, this definition gives the definition of the hamiltonian cycle in ordinary graphs for $r=2$.

Many questions about hamiltonian cycles involve some degree condition on the graph. The notion of the degree is also extended for hypergraphs, it is defined below in full generality, however, only some special cases will be used.

\footnotetext{
${ }^{\dagger}$ Research supported by OTKA grants OTKA T 046234 and T 043520

1365-8050 (C) 2005 Discrete Mathematics and Theoretical Computer Science (DMTCS), Nancy, France
} 
Definition 2 The degree of a fixed $l$-tuple of distinct vertices, $\left\{v_{1}, v_{2}, \ldots, v_{l}\right\}$, in a r-uniform hypergraph is the number of edges of the hypergraph containing the set $\left\{v_{1}, v_{2}, \ldots, v_{l}\right\}$. It is denoted by $d_{r}\left(v_{1}, v_{2}, \ldots, v_{l}\right)$. Furthermore $\delta_{r}^{(l)}(\mathcal{H})$ denotes the minimum of $d_{r}\left(v_{1}, v_{2}, \ldots, v_{l}\right)$ over all l-tuples of vertices in $\mathcal{H}$. The neighbourhood of a vertex $v$ is defined by

$$
N_{\mathcal{H}}(v):=\{E-\{v\} \mid v \in E, E \in \mathcal{E}(\mathcal{H})\}
$$

Questions about hamiltonian cycles are usually not easy. One reason of this is probably that the problem of deciding whether a given graph contains a hamiltonian cycle is an NP-complete problem. From this point of view we cannot expect the hypergraph versions of these questions to be easier, since the corresponding problem for Hamiltionian chain is also NP-complete for any fixed $r$. This was proved by Müller.

An other reason might be the following. In graphs, if there is a path from $v \in V(G)$ to $u \in V(G)$ and there is a path from $u \in V(G)$ to $w \in V(G)$, then there is a path from $v \in V(G)$ to $w \in V(G)$. This fact makes it possible to define components in graphs. However, the above claim does not hold in hypergraphs, so it is more complicated to find a good notion replacing "components".

In each of the following four Sections we will deal with a separate question about hamiltonian chains.

\section{Dirac type theorem}

Dirac (1952) proved that if the minimal degree of a graph on $n$ vertices is at least $\frac{n}{2}$ then it has a hamiltonian cycle (chain). This is tight, as it is demonstrated by the graph obtained from two disjoint nearly equal cliques and another vertex that is adjacent to every vertex in the union of the cliques. The following result attempts to generalise this result to hamiltonian chains in $r$-uniform hypergraphs $\mathcal{H}$ with large $l$-minimum degree.

Theorem 3 (Katona and Kierstead (1999)) If $\mathcal{H}=(V, \mathcal{E})$ is an $r$-uniform hypergraph on $n$ vertices with $\delta_{r-1}(\mathcal{H})>\left(1-\frac{1}{2 r}\right) n+4-r-\frac{5}{2 r}$, then $\mathcal{H}$ contains a hamiltonian chain.

The bound given in Theorem 3 is probably not best possible. However, it is quite easy to show that this bound cannot be lower than $\left\lfloor\frac{n-r+1}{2}\right\rfloor$.

A few years later Ruciński et al. proved that this bound is tight if $r=3$ and $n$ is sufficiently large. Since the proof uses the hypergraph version of Szemerédi's regularity lemma, sufficiently large here means extremely large.

Recently the same authors announced that they can prove the above result for general $r$ and also they improved the lower bound on $n$, but is still huge. Therefore this question is seems to be more or less settled, but it would be still nice to have a proof for "normal size" hypergraphs, too.

\section{$3 \quad k$-edge-hamiltonian hypergraphs}

Definition 4 A hypergraph is hamiltonian if it contains a hamiltonian-chain and it is $k$-edge-hamiltonian if by the removal of any $k$ edges a hamiltonian hypergraph is obtained.

The main aim of the present section is to investigate minimum size $k$-edge-hamiltonian hypergraphs. This was already settled for graphs: 
Theorem 5 (Paoli et al. (1986); Wong and Wong (1984)) The number of edges in a minimum k-edgehamiltonian graph on $n \geq k+3$ vertices is $\lceil n(k+2) / 2\rceil$.

Since the degree of any vertex in an $r$-uniform hamiltonian chain is $r$, the minimum degree in a $k$-edgehamiltonian hypergraph is at least $r+k$, so the number of edges is at least $\lceil n(r+k) / r\rceil$. For $r=2$ this shows that the constructions in the above theorem are best possible. However, for $r>2$ this lower bound is not best possible.

We establish some upper and lower bounds for some special cases of $r$ and $k$.

If a hypergraph contains $k+1$ edge-disjoint hamiltonian chains, then it is clearly $k$-edge-hamiltonian. This observation leads to the trivial upper bound on the minimum number of edges: $(k+1) n$. If $k=1$ and $r=3$ then the following slightly better upper bound is obtained.

Theorem 6 (Frankl and Katona) There exists a 1-edge-hamiltonian 3-uniform hypergraph $\mathcal{H}$ on $n$ vertices with

$$
|\mathcal{E}(\mathcal{H})|=\frac{11}{6} n+o(n) .
$$

Theorem 7 (Frankl and Katona) For any 1-edge-hamiltonian 3-uniform hypergraph $\mathcal{H}$ on $n \geq 5$ vertices

$$
|\mathcal{E}(\mathcal{H})| \geq \frac{14}{9} n
$$

holds.

The proof uses some observations about the structure of the neighbourhood of the vertices. It is important to note, that in a 3 -uniform hypergraph the neighbourhood of a vertex is not just a set of vertices, but a (2-uniform) graph. In general, the neighbourhood of a vertex in an $r$-uniform hypergraph is an $(r-1)$ uniform hypergraph. The first important observation in the proof is that if there is a hamiltonian chain in a hypergraph, then the neighbourhood of any vertex must contain an $(r-1)$-uniform chain of length $2 r-3$.

Using similar ideas the authors prove some other special cases, too.

Theorem 8 (Frankl and Katona) There exists a 2-edge-hamiltonian 3-uniform hypergraph $\mathcal{H}$ on $n$ vertices with

$$
|\mathcal{E}(\mathcal{H})|=\frac{13}{4} n+o(n) .
$$

Theorem 9 (Frankl and Katona) For any 2-edge-hamiltonian 3-uniform hypergraph $\mathcal{H}$ on $n$ vertices

$$
|\mathcal{E}(\mathcal{H})| \geq 2 n
$$

holds.

Now we state lower and upper bounds for $r>3$.

Theorem 10 (Frankl and Katona) There exists a 1-edge-hamiltonian $r$-uniform hypergraph $\mathcal{H}$ on $n$ vertices with

$$
|\mathcal{E}(\mathcal{H})|=\frac{4 r-1}{2 r} n+o(n) .
$$


Theorem 11 (Frankl and Katona) For any 1-edge-hamiltonian 4-uniform hypergraph $\mathcal{H}$ on $n \geq 6$ vertices

$$
|\mathcal{E}(\mathcal{H})| \geq \frac{3}{2} n
$$

holds.

The question raised in this section is still open even for the spacial cases. It looks difficult to find the exact constant factor already for the $r=3, k=1$ case.

\section{Maximally non-hamiltonian hypergraphs}

What is the maximum number of edges in an r-uniform hypergraph on $n$ vertices which has no hamiltonian chain?

Ore (1961) proved that for graphs there is a unique maximal graph $G$ with $\left(\begin{array}{c}n-1 \\ 2\end{array}\right)+1$ edges. The graph $G$ is formed from a $(n-1)$-clique together with one additional edge incident to the remaining vertex.

First Katona and Kierstead (1999) gave an easy lower bound, but later this was slightly improved in Frankl and Katona.

Theorem 12 (Frankl and Katona) If an $r$-uniform hypergraph $\mathcal{H}$ on $n$ vertices has no hamiltonian chain then

$$
|\mathcal{E}(\mathcal{H})| \leq\left(1-\frac{4 r}{(4 r-1) n}\right)\left(\begin{array}{l}
n \\
r
\end{array}\right)
$$

holds.

The best known construction is due to Tuza.

Theorem 13 (Tuza) For any $n>r$ there exists an $r$-uniform hypergraph on $n$ vertices with

$$
\left(\begin{array}{c}
n-1 \\
r
\end{array}\right)+\left(\begin{array}{l}
n-1 \\
r-2
\end{array}\right)+o(n)
$$

edges.

\section{Hamiltonian chain saturated hypergraphs}

Definition 14 We say that hypergraph $\mathcal{H}$ is hamiltonian chain saturated if $\mathcal{H}$ does not contain a hamiltonian chain but by adding any new edge we create a hamiltonian chain in $\mathcal{H}$. Similarly, we say that $\mathcal{H}$ is hamiltonian path saturated if $\mathcal{H}$ does not contain an open hamiltonian chain, but by adding any new edge we create an open hamiltonian chain in $\mathcal{H}$.

The question investigated in this section is the following: What is the minimum number of edges in a hamiltonian path (chain) saturated hypergraph on $n$ vertices?

We will only deal now with the hamiltonian path saturated case. For graphs, usually the questions for hamiltonian cycle and path have similar solutions. If we have an answer for one of the questions, the other one follows easily. So although we deal now with the path case only, hopefully similar results can be proved for the chain case. However, it is important to note, that if $r>2$ than an open hamiltonian chain and a hamiltonian chain is "not so close" then in the graph case. One can create a hamiltonian cycle 
from a hamiltonian path by adding a single edge. On the other hand, in the general case $r-1$ edges are needed to achieve the same goal.

The above question for graphs was raised by Bollobás (1978) and solved completely for hamiltonian cycles in a series of papers by several authors.

The corresponding results for the hamiltonian path saturated case can be found in Dudek et al.. These results are nearly sharp. The construction are based on Isaak's snarks and generalized Petersen graphs, which looks to be impossible to generalise for hypergraphs.

First we prove a lower bound on the number of edges for the 3-uniform hamiltonian path saturated case.

Theorem 15 (Dudek and Katona) If $\mathcal{H}$ an $r$-uniform hypergraph is hamiltonian chain saturated, then $|\mathcal{E}(\mathcal{H})| \geq\left(\begin{array}{l}n \\ r\end{array}\right) /(r(n-r)+1)$.

The best construction so far has more edges then this. Even the order of magnitude is larger.

Theorem 16 For any $n \geq 7$ there exists a 3-uniform hypergraph with $\frac{2}{27} n^{3}+o\left(n^{3}\right)$ edges.

The authors conjecture that the right order of magnitude is $n^{r-1}$ in the general case. This question and the corresponding question for the hamiltonian chain saturated case is still open.

\section{References}

B. Bollobás. Extremal graph theory, volume 11 of London Mathematical Society Monographs. Academic Press Inc. [Harcourt Brace Jovanovich Publishers], London, 1978. ISBN 0-12-111750-2.

G. A. Dirac. Some theorems on abstract graphs. Proc. London Math. Soc. (3), 2:69-81, 1952.

A. Dudek and G. Y. Katona. The generalization of zelinka constructions for 3-uniform hypergraphs. manuscript.

A. Dudek, G. Y. Katona, and A. P. Wojda. Hamiltonian path saturated graphs with small size. to appear in Discrete Appl. Math.

P. Frankl and G. Y. Katona. k-edge-hamiltonian hypergraphs. submitted to J. of Graphs Theory in 2002.

G. Y. Katona and H. A. Kierstead. Hamiltonian chains in hypergraphs. J. Graph Theory, 30(3):205-212, 1999. ISSN 0364-9024.

H. Müller. privat communication. 1998.

Ø. Ore. Arc coverings of graphs. Ann. Mat. Pura Appl. (4), 55:315-321, 1961.

M. Paoli, W. W. Wong, and C. K. Wong. Minimum $k$-Hamiltonian graphs. II. J. Graph Theory, 10(1): 79-95, 1986. ISSN 0364-9024.

A. Ruciński, V. Rödl, and E. Szemerédi. privat communication. 2001.

Z. Tuza. private communication.

W. W. Wong and C. K. Wong. Minimum k-Hamiltonian graphs. J. Graph Theory, 8(1):155-165, 1984. ISSN 0364-9024. 
